Methods Studies were performed on 125 infants $<29$ weeks gestation who required mechanical ventilation at 7-14 days and received 24 days of iNO at 20-2 ppm. A control group of 19 infants did not receive iNO.

Results In NO-treated infants there was a significant dose-dependent increase of both urinary NOx and cGMP per creatinine (maximal 3.1- and 2-fold, respectively, at 10-20 ppm iNO) compared to off iNO. The ratio of cGMP to NOx, which may reflect efficiency of NO signalling via guanylate cyclase, was lower (mean 38\%) at all doses of iNO compared to off NO. NOx and cGMP concentrations at both $2 \mathrm{ppm}$ and off iNO were inversely related to severity of lung disease (Respiratory Severity Score) during the first month, and the NOx levels were lower in infants who died or developed BPD at term. NOx was higher in Caucasian compared to other infants at all iNO doses.

Conclusions Urinary NOx and cGMP are biomarkers of endogenous NO production and lung uptake of iNO, and levels at some doses reflect the severity of lung disease in infants. These results support a role of the NO-cGMP pathway in lung development and repair from injury.

\section{PS-206 MATERNAL/NEONATAL VITAMIN D DEFICIENCY MAY BE A RISK FACTOR FOR DEVELOPMENT OF BRONCHOPULMONARY DYSPLASIA IN PRETERM INFANTS}

${ }^{1} \mathrm{M}$ Cetinkaya, ${ }^{1} \mathrm{~A}$ Demirhan, ${ }^{1} \mathrm{G}$ Buyukkale, ${ }^{2} \mathrm{~F}$ Cekmez, ${ }^{1} \mathrm{~T}$ Erener-Ercan, ${ }^{2} \mathrm{G}$ Aydemir, ${ }^{3} \mathrm{FN}$ Aydin, ${ }^{4} \mathrm{~T}$ Tunc. ${ }^{1}$ Neonatology, Kanuni Sultan Suleyman Training and Research Hospital, Istanbul, Turkey; ${ }^{2}$ Neonatology, Gulhane Military Medical Academy, Istanbul, Turkey; ${ }^{3}$ Biochemistry, Gulhane Military Medical Academy, Ankara, Turkey; ${ }^{4}$ Neonatology, Gulhane Military Medical Academy, Ankara, Turkey

\subsection{6/archdischild-2014-307384.504}

Background and aims Vitamin D seems to play an important role in the pathogenesis of respiratory system diseases. The aim of this study was to evaluate the possible association between both maternal and neonatal ${ }^{2} 5$-hydroxyvitamin-D (25-OHD) levels and the subsequent risk of bronchopulmonary dysplasia (BPD)development in preterm infants.

Methods Premature infants $\leq 32$ gestational age and admitted to Neonatal Intensive Care Unit with a diagnosis of respiratory distress syndrome (RDS) between December 2012 and December2013 were included to this prospective study. Blood for neonatal and maternal vitamin D levels were obtained from all infants and their mothers at the time of hospital admission. The maternal and neonatal demographic features, maternal vitamin D usage, maternal head cover status, birth season and neonatal morbidities and mortality were all recorded.

Results A total of 100 preterm infants were included and 31 of them developed BPD. The mean birthweight, gestational age, duration of ventilation and duration of oxygen supplementation were significantly higher in infants with BPD compared with those who did not develop BPD ( $\mathrm{p}<0.05)$. Both maternal (19 \pm 2.2 vs $28.7 \pm 7.6)$ and neonatal $(7.1 \pm 1.6$ vs $14.8 \pm 4.7)$ 25-OHD levels were significantly lower infants with BPD (both $\mathrm{p}>0.05)$. All of the infants with BPD had a 25-OHD level $<10 \mathrm{ng} / \mathrm{ml}$ that represented severe vitamin D deficiency $(\mathrm{p}<$ $0.05)$.

Conclusions This study suggested for the first time that maternal/neonatal vitaminD deficiency might be associated with increased risk of BPD in preterm infants.

\section{PS-207 PLASMA PRO-ENDOTHELIN-1 AS MARKER OF BRONCHOPULMONARY DYSPLASIA}

${ }^{1} \mathrm{~S}$ Wellmann, ${ }^{1} \mathrm{I}$ Pramana, ${ }^{2} \mathrm{~B}$ Grass, ${ }^{3} \mathrm{~K}$ Spanaus, ${ }^{4} \mathrm{~S}$ Fouzas, ${ }^{5} \mathrm{C}$ Bührer, ${ }^{6} \mathrm{P}$ Baumann. ${ }^{1}$ Neonatology, University Children's Hospital Both Basel, Basel, Switzerland; 'Intensive Care Medicine and Neonatology, University Children's Hospital Zurich, Zurich, Switzerland; ${ }^{3}$ Institute of Clinical Chemistry, University Hospital Zurich, Zurich, Switzerland; ${ }^{4}$ Neonatology, University Hospital of Patras, Patras, Greece; ${ }^{5}$ Neonatology, Charité University Medical Center, Berlin, Germany; ${ }^{6}$ Neonatology, University Hospital Zurich, Zurich, Switzerland

\subsection{6/archdischild-2014-307384.505}

Background Endothelin-1 (ET-1) is a potent pulmonary vasoconstrictor, involved in lung injury and remodelling. ET-1 can be estimated by measuring its stable by-product, C-terminal pro-ET1 (CT-proET-1), in plasma.

Aims To investigate CT-proET-1 values in very preterm infants (gestational age $<32$ weeks) from birth to postmenstrual age (PMA) of 36 weeks, and their relationship with lung injury and bronchopulmonary dysplasia (BPD).

Methods Prospective cross-sectional study of 391 CT-proET-1 measurements (fully automated immunoflorescent assay) from 267 very preterm infants. Measurements were performed at birth ( $\mathrm{n}=72$ infants), on day of life (DOL) $2(\mathrm{n}=89)$, on DOL $6(\mathrm{n}=49)$, on DOL $28(\mathrm{n}=106)$, and at PMA $36(\mathrm{n}=$ 75). Trial registration: ClinicalTrials.gov NCT01644981.

Results CT-proET-1 values were (median) $151 \mathrm{pmol} / \mathrm{L}$ (IQR 118-186) at birth, peaked on DOL 2 (319 pmol/L (235-382)), and declined thereafter to $214 \mathrm{pmol} / \mathrm{L}(148-293)$ on DOL 6, $184 \mathrm{pmol} / \mathrm{L}$ (149-233) on DOL 28, and $150 \mathrm{pmol} / \mathrm{L}$ (118-188) at PMA 36. Infants with BPD had higher CT-proET-1 values on DOL $2(\mathrm{p}=0.001)$, DOL $6(\mathrm{p}<0.001)$, and DOL $28(\mathrm{p}=$ 0.007 ), with no differences at birth and PMA 36 as compared to those without BPD. CT-proET-1 on DOL 6 was significantly correlated with days of mechanical ventilation, nasal CPAP, and oxygen requirement (Spearman's Rs 0.657, 0.713, 0.745, respectively, $\mathrm{p}<0.001$ each). Moderate correlations were found for the same parameters on DOL 2 and DOL 28 but not for birth and PMA 36.

Conclusions The levels and the pattern of CT-proET-1 increase during the first week of life might serve as an early marker of BPD.

\section{PS-208 GENETIC PREDISPOSITION TO THE DEVELOPMENT OF BRONCHOPULMONARY DYSPLASIA IN INFANTS BORN PREMATURELY}

${ }^{1}$ CA May, ${ }^{2} \mathrm{~S}$ Best, ${ }^{3}$ J Peacock, ${ }^{4} \mathrm{SL}$ Thein, ${ }^{5} \mathrm{~A}$ Greenough. ${ }^{1}$ The Royal London Neonatal Unit, Barts Health NHS Trust, London, UK; ${ }^{2}$ Molecular Haematology, King's College Hospital NHS Trust, London, UK; ${ }^{3}$ Medical Statistics, King's College London, London, UK; ${ }^{4}$ Molecular Haematology, King's College London, London, UK, ${ }^{5}$ Asthma Allergy and Lung Biology, King's College London, London, UK

\subsection{6/archdischild-2014-307384.506}

Background and aims Bronchopulmonary dysplasia (BPD) is an unfortunately common outcome following premature birth. Genetic factors influence BPD development but their role is part of a complex interaction with environmental factors. We postulated that alterations in the gene as well as imbalances in gene products may affect BPD development.

Methods The NIH human genome database was interrogated for previously identified gene polymorphisms that have been associated with neonatal respiratory conditions. Angiotensin converting enzyme (ACE) and surfactant proteins A-D gene candidates were selected based upon clinical plausibility for the study 